\title{
Does cancer research have realistic aims?
}

\begin{abstract}
The Imperial Cancer Research Fund tries with its 1973 report, recently published, to appeal to a wider audience. Brian Ford takes a look at the report and at broader issues in cancer research.
\end{abstract}

CANCER research is a blend, not altogether a happy one, of the assured with the uncertain, the esoteric with the mundane; yet in recent years some remarkable improvements in survival rates have been achieved in fields that include breast cancer, leukaemia and the lymphomas. That alone is sufficient to refute allegations that this speciality is uniquely able to consume public funding for little tangible benefit. Successes in specific areas are, however, not themselves a guarantee that priorities are correct or that courses of action have been chosen for valid criteria. The latest report of the Imperial Cancer Research Fund (Lincoln's Inn Fields, London WC2) throws an interesting light on the advancing front of progress, and mirrors some deficiencies in the philosophy that underlies it.

Past reports have been issued by the fund in a different form. As the Foreword acknowledges, they have been an unsatisfactory mixture of facts, figures and data presented (particularly where clinical findings and research are concerned) in the specialist form least likely to attract interest or even understanding from the outside reader. The survey for 1973 is therefore novel in approach, for it embraces three specific and distinct areas of interest: the accounts (which are bound as a separate volume), the research reports as such and the Director's assessment. There has been a deliberate attempt to word this introductory section carefully so that it may be assimilable to the intelligent outsider (who is described as a 'non-scientist' by the Foreword, but in this era of isolationist specialisation is as likely to be a psychologist or physicist) and Michael Stoker has taken the brave step of using his easy manner and flow of commonplace English to accomplish the task. For so long has it been widely assumed that understandable terminology and uncomplicated syntax are indicators of superficiality in approach, that this step is perhaps doubly notable. Yet in some respects, by casting a more direct light on the state of the art, it is a less than satisfactory exercise.

He draws a close parallel between the immune response that may be invoked against malignant cells and that seem to act "against measles". Similarly far-fetched analogies are becoming popular (indeed the Nature-Times News Service report dated April 22, 1974, draws a similar parallel) yet it is arguably unrealistic. Surely the infective invasion of susceptible tissues by a transmissable viral genome is operationally distinct from neoplastic transformation, and the quest for a genotypically polyvalent vaccine, as it were, must be a different kettle of fish from the development of measles immunisation. One looks back to the trial-and-error attempts of Jenner to experiment with vaccinia and Pasteur with rabies, both by good fortune working with material which responded uniquely to their techniques though neither had much reason to suppose it would do so; and one assumes there is something more predictable, less metaphysical, about modern medical methodology. Yet here we are, in the 1970s, with electroshock in the psychiatric hospitals and vaccineshock in oncology, hopefully injecting BCG or irradiated leukaemic blast cells, in the hope of gingering up the immunological sensitivities of a cancer patient.

In Stoker's words it looks sound enough: "The vaccine is made from leukaemic blood from another patient, which is slightly different from the recipient's own blood and may, therefore, give an additional boost to the defences". Yet it is this very clarity which reveals the extent to which our

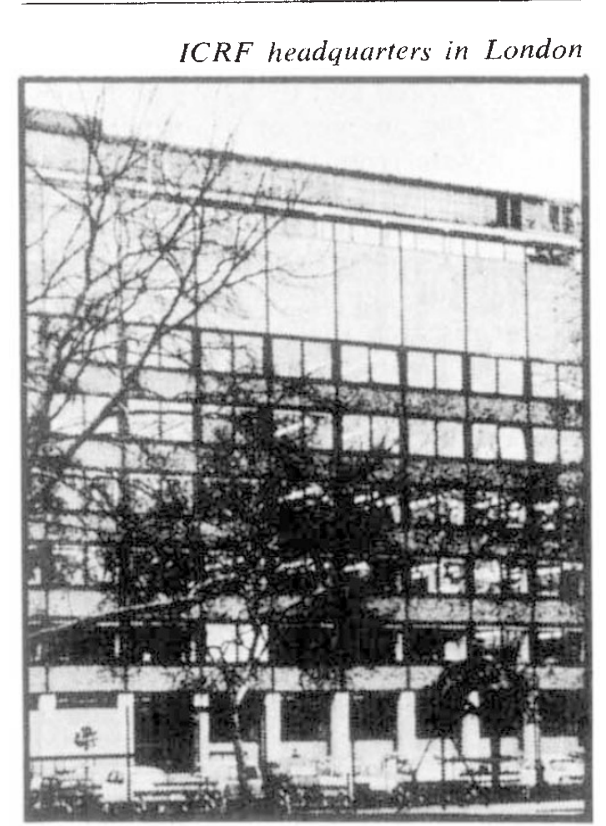

knowledge is clouded and incomplete.

There are many other fields of investigation in which the dearth of detailed knowledge, and the inadequacy of many orthodox beliefs, is demonstrated. The report mentions that Rubens and Dulbecco have investigated the use of cytotoxic compounds adsorbed onto tumour-specific antibodies, the principle being to utilise the antibody as a vehicle for the drug, only to find that the loose covalent association that was assumed did not, apparently, occur in their trials. It now seems that the increase in susceptibility of the transformed cells is coincidentally induced by the presence of antibody; how remains uncertain.

Though some of the practical problems of cancer management remain poorly understood, the study of what we might in the broad sense term oncogenetics is inspiring. So too is the interest in conceptualising about cell growth. I believe it is helpful to construct greater models by which to account for the lesser datum; holistic concepts through which to marry reductionist dogmas. In this respect it is heartening to see the work which Martin and his colleagues have under way, which construct novel interpretations of the dynamic status of mature cell communities; models that are not profound and whose principal benefit lies in their being far from complex, but which provide an intellectual synthesis of observed phenomena.

Growth-rate regulatory mechanisms are what cancer is all about, and it is fitting that one of the leading ICRF groups should be the Department of Cell Regulation. Yet, when our understanding of these mechanisms is so profoundly complex in some respects, how surprising it is that Stoker can demonstrate the simple fact that boundary layer gradients may be as important as cell-to-cell contact in regulating cell growth rates. This contrast between the detail of our understanding in some respects, and our ignorance of far more fundamental, practical issues in others, can be illustrated by other reports in the document. Though it is now beginning to seem possible that we may be able to screen urine samples as a means of detecting those patients peculiarly susceptible to breast cancer, it is still not known whether radical mastectomy or wide excision are preferable in the early stages of the disease. Here too we see progressive developments in one aspect of a discipline 
Research in progress at St Bartholomew's Hospital, London, where the Imperial Cancer Research Fund established a Professorial Chair in Medical Oncology in 1971.

contrasting with ignorance of a seemingly elementary nature in another.

In certain respects the sense of imbalance that parts of the ICRF report engender are no more than a mirror of the greater problems faced by modern science. To rely, in some primitive Baconian sense, on observing phenomena and then adopting the correctly self-critical mode advocated by the followers of Professor Popper, seems to be the only true path to objectivity and insight. Too often it tends, instead, to blind one to the true relationships between the larger fragments of science, and it conveniently ignores the weaknesses and foibles of human nature. I believe we should now recognise the inability of modern scientific research to fulfil its function adequately by relying solely on what one might term inspirational research. Perhaps we need something of what the Americans would term the 'project' approach, in which teams or individuals are asked to prosecute a given line of investigation which fitted into the whole.

The argument against this is that in this way we tend towards the dictatorial direction of research. What we seem to forget is that research is being surely directed at the moment, but directed by whim, by fancy, by inclination; it is directed by current trends, by the concensus paradigm subconsciously obeyed by an introspective discipline of science; and it is directed by the distribution of funds and support. Though there is no need to dictate science policy, or to set the sights of science at some invisible target, there is certainly room for a little calculated realism somewhere in the mix.

Even though we pay little attention to the criteria by which our research priorities are selected, they certainly exist. To utilise a framework of agreed values to supplement-though in no sense replace-'personal fulfilment' guidelines is more likely to bring us nearer the truth than shots in the dark, no matter how inspired the marksman. Though it is undeniably true that free-wheeling

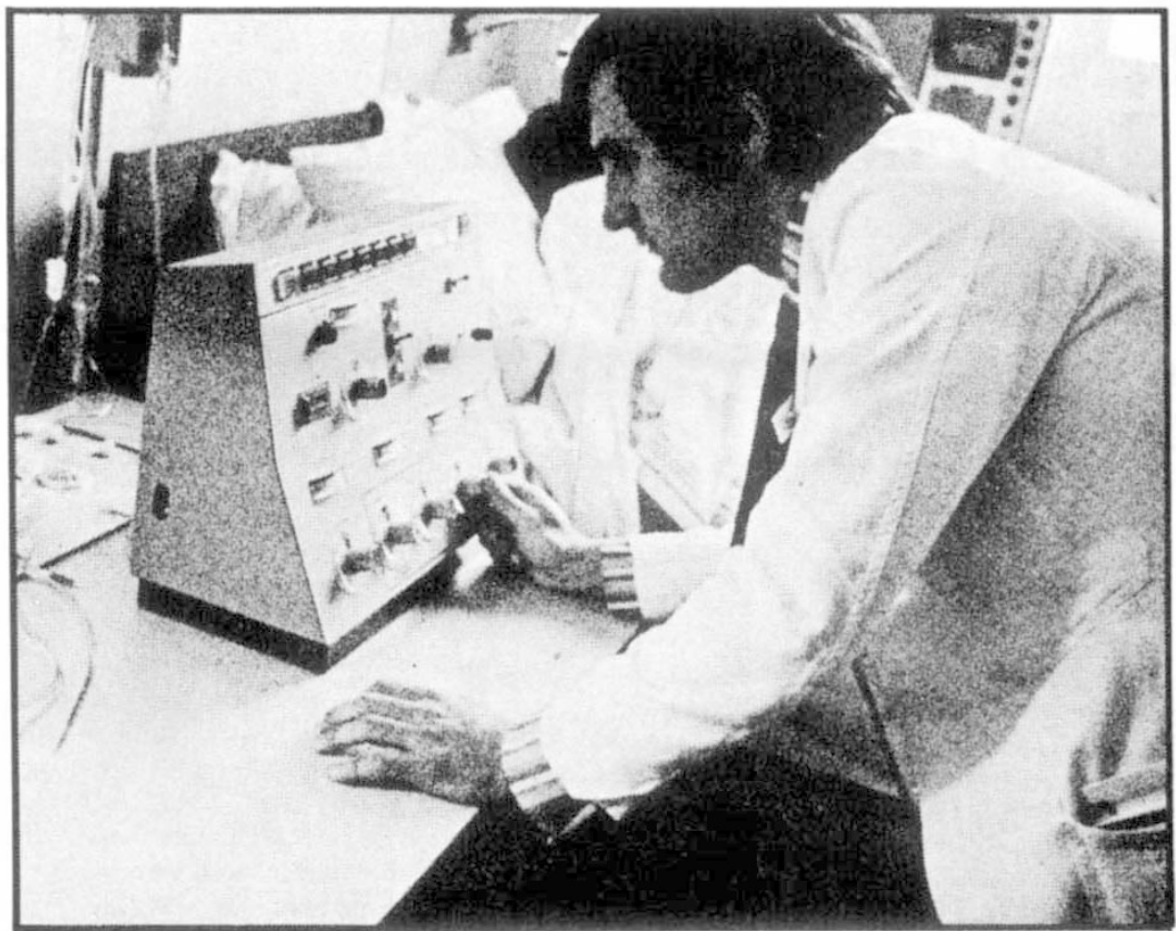

chance gave us Penicillium notatum and the earlier discovery of antibiosis, it was the directed research of wartime that gave the world penicillin and the antibiotic age. In an area such as cancer research, where the main concern of all is to bring about an increase in knowledge, unless they or their relatives are suffering from the disease, when the prime requirement is seen as the saving of life; here more than anywhere we need guidance by concensus and objective-setting as a supplement to inspiration.

To speak of cost-effectiveness in research is irksome--until we realise how costly it is, and how badly civilisation needs the effects.

If we have to pick out one example of an individual revelation from the ICRF report, there can be no better example than the discovery of a demonstrable difference between the cell surface in normal and transformed tissues, namely the absence of a normally-present protein from the surface of malignant cells. This has been the work of Richard Hynes in the Department of Tumour Virology, and is a sound and hopeful advance. Indeed it is in virological research that the ICRF has perhaps its greatest claim to fame, and around which at least four of the groups base their work. Yet man seems to show few signs of suffering from virusinduced tumours, and current estimates suggest that $60-90 \%$ of all malignancies in man are caused by chemical carcinogens. Should one suggest that-if resources are limited-more attention should be paid to the practical problems of patient care in hospitals and at home?

We still know little about cells in communities. A model of histological or developmental normality-cell sociology, one might say-is at least as important in understanding malignancy as would be a comprehensive understanding of a single cell. If we are to promulgate pure research, ought it not to concern itself with relatively unexplored avenues such as these?

It is often said that in cancer one cannot experiment on humans. But one does, of course, perpetually in a manner which (though entirely justifiable and ethical) must surely seem incongruous alongside specialised research in other fields. How is it there is still the scent of alchemy in the air where much chemotherapy is concerned, utilising drugs of high potency but uncertain action in an empirical and almost haphazard manner? Should the arm be immobilised after breast surgery or allowed freedom of movement as an aid to fluid drainage? Are steroids helpful in the therapy of malignant disease? These questions (which seem fundamental indeed alongside specialised virus research) are only now being resolved, by experimental trial.

When there are many short term, immediate questions to be answered, is it entirely justifiable to press ahead in such detail on animal research based on cancer models that are not apparent in mankind? Perhaps we should take heed of the comments in last year's report from the International Agency for Research on Cancer in Lyon concerning the near impossibility of extrapolating the mass of data from animals to man. Indeed, is not the problem of cancer research that we know too much: we have too many data and too few conceptual pegs on which to hang them? And is the balance of cancer research cntirely fair to the patient? 